\title{
Clinical Study \\ Efficiency and Safety of Prolonged Levosimendan Infusion in Patients with Acute Heart Failure
}

\author{
Georgios Aidonidis, ${ }^{1,2}$ Ioannis Kanonidis, ${ }^{2}$ Vasileios Koutsimanis, ${ }^{2}$ Till Neumann, ${ }^{1}$ \\ Raimund Erbel, ${ }^{1}$ and Georgios Sakadamis ${ }^{2}$ \\ ${ }^{1}$ Department of Cardiology, West German Heart Center, Medical School, University of Duisburg-Essen, 45122 Essen, Germany \\ ${ }^{2}$ 2nd Department of Cardiology, Hippokration General Hospital, Medical School, Aristotle University of Thessaloniki, \\ 54642 Thessalloniki, Greece
}

Correspondence should be addressed to Georgios Aidonidis, georgaidonis@yahoo.gr

Received 1 November 2010; Revised 10 January 2011; Accepted 3 February 2011

Academic Editor: Gregory Giamouzis

Copyright ( $\odot 2011$ Georgios Aidonidis et al. This is an open access article distributed under the Creative Commons Attribution License, which permits unrestricted use, distribution, and reproduction in any medium, provided the original work is properly cited.

\begin{abstract}
Background. Levosimendan is an inotropic drug with unique pharmacological advantages in patients with acute heart failure. Scope of this study is to determine whether longer infusion patterns without the hypotension-inducing loading dose could justify an effective and safe alternative approach. Methods. 70 patients admitted to the emergencies with decompensated chronic heart failure received intravenously levosimendan without a loading dose up to 72 hours. Clinical parameters, BNP (Brain Natriuretic Peptide) and signal-averaged-ECG data (SAECG) were recorded up to 72 hours. Results. The 48-hour group demonstrated a statistically significant BNP decrease $(P<.001)$ after 48 hours, which also maintained after 72 hours. The 72-hour group demonstrated a bordeline decrease of BNP after 48 hours $(P=.039)$, necessitating an additional 24-hour infusion to achieve significant reduction after 72 hours $(P<.004)$. SAECG data demonstrated a statistically significant decrease after 72 hours $(P<.04)$. Apart from two deaths due to advanced heart failure, no major complications were observed. Conclusion. Prolonged infusion of levosimendan without a loading dose is associated with an acceptable clinical and neurohumoral response.
\end{abstract}

\section{Introduction}

Levosimendan is recognized as an inotropic drug used in acute or decompensated chronic heart failure with innovative characteristics. It is not a $\beta$-adrenergic agonist which would have energy-consuming and proarrhythmic effects. It is rather a mild PDE inhibitor in clinical relevant doses [1] and mainly a Ca-dependant troponine-I sensitizer resulting in energetically beneficial contractility of the cardiac muscle. Furthermore, there is also an ATP-dependant Kchannel activation which causes peripheral vasodilation [2]. A cardioprotective mechanism via the ATP-K channels and the phenomenon of preconditioning is also under research. Pharmacokinetically, levosimendan acts for prolonged time, since its two major metabolites OR-1855 and OR-1896 have half-life time of $70-80$ hours [3]. This pharmacological profile offers an ideal medical option in acute heart failure with preserved or borderline systolic blood pressure [4]. Standard pattern of infusion consists of a loading dose and a continuous 24-hour i.v. infusion. In some cases, the vasodilatory action causes an early hypotension resulting in withdrawal of drug or coadministration with a $\beta$-agonist with all the possible risks of this combination [5]. The goal of this observational study was primarily to determine whether a prolonged and beyond the 24-hour infusion pattern without the loading dose could be efficient and safe for patients in acute or decompensated heart failure. For this reason, we administrated a prolonged levosimendan infusion for 24,48 , or 72 hours creating three subgroups.

Clinical and neurohormonal responses were measured with simple bedside parameters derived from physical examination [6] and serial measurements of BNP [7] and SAECG. Safety was determined by the presence of major or minor complications and onset of new arrhythmias [8]. 


\section{Methods}

2.1. Patients. Study population consisted of patients admitted to hospital between March 2003 and December 2006 suffering from acute heart failure or decompensated chronic heart failure. The clinical status of those patients was III or IV according to the NYHA classification. All patients were resistant to optimal medical therapy and did need support of an inotropic agent. Diagnosis of heart failure was confirmed by the contribution of physical examination, previous history, but mainly by transthoracic echocardiography and thoracic X-rays. Ejection fraction less than $45 \%$ in echocardiogram was documented in all patients. Retrospective analysis of the heart failure aetiology was performed.

As for the exclusion criteria, patients with an acute coronary syndrome, cardiac shock with systolic blood pressure under $85 \mathrm{mmHg}$ resistant to volume administration, hypertrophic cardiomyopathy, benign or life-threatening tachyarrhythmias with heart rate over $120 / \mathrm{min}$, and electrolytic abnormalities did not participate.

2.2. Study Protocol. The study protocol included quantitative analysis in three different subgroups of main population determined by clinical evaluation after 24-hour intervals. All participants took next to their standard medication (diuretics, oxygen, ACEs or ARBs, digoxin, and $\beta$-blockers in minor dose) an infusion of levosimendan of $0.05 \mu \mathrm{g} / \mathrm{min} / \mathrm{Kg}$ uptitrated in two hours to $0.1 \mu \mathrm{g} / \mathrm{min} / \mathrm{Kg}$ for 24 hours. In the interval, if there was no contraindication, the uptitration could achieve the dose of $0.2 \mu \mathrm{g} / \mathrm{min} / \mathrm{Kg}$.

24-hour and 48-hour time points after initial infusion were crucial. It should be decided if levosimendan could be stopped or continued according to bedside clinical criteria. The subjective criterion of patient's well-being and the absence of pathologic signs on auscultation of the pneumonal areas or the absence of S3 gallop were cut points for the discontinuation of levosimendan infusion. Patients, who continued the infusion, did so for a duration of 72 hours. The exclusion criteria of recruitment had the same power during the infusion time, so that management could be safe. In some complications, like hypotension or tachyarrhythmia, it was up to investigator to treat the complication and to decide for the next step of the study.

2.3. Measurements. All patients were measured for several countable variables. Blood pressure and heart rate were determined at baseline and at 24,48 , and 72 hours, respectively.

A resting ECG was performed at the above-mentioned time points confirming also the possible arrhythmogenic complications. In cases a confirmation of heart failure was needed, a transthoracic echo was performed.

As far for the neurohormonal response of the therapy, we used measurements of brain natriuretic peptide (BNP) by means of the microspheric ELISA analysis (MEIA) method. Blood samples of $6 \mathrm{~mL}$ were taken at the beginning time point of the infusion, at 48 hours and at 72 hours independently on the subgroup a patient belonged to.
After an addition of $0.1 \mathrm{~mL}$ transylol for preserving protein molecules, these samples were centrifuged at 3000 cycles/ min for 5 minutes. The serum taken was frozen on $-20^{\circ}$ Celsius for retrospective BNP measurements. BNP normal range was assumed under the value of $100 \mathrm{pg} / \mathrm{mL}$.

On the study, a signal averaged ECG was used (Marquette model 5000). P-wave filtered, QRS filtered duration, root mean square voltage, the last $40 \mathrm{msec}$, and the duration of voltage $<40 \mu \mathrm{V}$ at $40 \mathrm{~Hz}$ were determined at baseline, 48 and 72 hours, respectively. Normal ranges of the above mentioned variables are QRS filtered duration $<120 \mathrm{msec}$, RMS the last $40 \mathrm{msec}>20 \mu \mathrm{V}$, and duration of low voltage at $40 \mathrm{~Hz}<38 \mathrm{msec}$.

Confirming an assumption of BNP response, we decided to determine two cut points. Relative BNP decrease of $>60 \%$ was evaluated as a good neurohormonal response.

BNP decrease $<20 \%$ or increase was evaluated as no response. The values between the two cut points were characterized as moderate responses.

Changes in QRS-filtered duration observed in this clinical study were evaluated with the assumption that variations in QRS complex duration could represent respective variations in left ventricular dimensions. So, decreases in QRSfiltered duration $>10$ msec were regarded as good response. Increases in QRS-filtered duration were evaluated as no response and the values in between as moderate ones.

The well-being status was evaluated by means of clinical examination and subjective confirmation of the patient at 24 and 48 hours of infusion. This semicountable variable was the determinator of continuation of levosimendan treatment.

Major complications were confirmed for the whole duration of the study and a period of a month followed. A continuous ECG monitoring during the infusion protocol confirmed the arrhythmias observed.

2.4. Statistics. Countable variables were demonstrated with minimum, maximum, mean, and standard deviation values. Differences in countable variables were evaluated with the nonparametric paired $t$-test of Wilcoxon.

Correlation between countable variables was evaluated with linear regression analysis. To confirm a comparison between the subgroups, we used the Kolmogorov-Smirnov and the Mann-Whitney tests.

Uncountable variables were evaluated in a descriptive manner and the correlation between them with the Chisquare test accompanied by the Phi or Cramer's V test. In all the tests the null hypothesis is rejected at confidence level of 5\%. SPSS.12 version statistical package was used.

2.5. Ethical Considerations. The protocol was approved by the Institutional Review Board of our center and was performed in accordance with institutional guidelines and the Declaration of Helsinki. All patients gave written informed consent before entering the study. 
TABLE 1: Demographic statistics at baseline of the study population.

\begin{tabular}{lcc}
\hline & Mean \pm St. dv. & Range \\
\hline Age (years) & $63.7 \pm 1.4$ & {$[36-88]$} \\
Mean arterial pressure $(\mathrm{mmHg})$ & $87.8 \pm 15.1$ & {$[72-126]$} \\
Heart rate $\left(\mathrm{min}^{-1}\right)$ & $76.5 \pm 10.5$ & {$[56-100]$} \\
BNP $(\mathrm{pg} / \mathrm{mL})$ & $1104 \pm 124$ & {$[51-4000]$} \\
Ejection fraction $(\%)$ & $33.5 \pm 6.3$ & {$[22-43.3]$} \\
$P$-wave duration $(\mathrm{msec})$ & $180 \pm 10$ & {$[78-322]$} \\
QRS filtered duration $(\mathrm{msec})$ & $149 \pm 4.1$ & {$[71-265]$} \\
\hline
\end{tabular}

Data are presented as Mean \pm Standard deviation.

TABLE 2: BNP and QRS filtered duration variations after 72 hours.

\begin{tabular}{|c|c|c|c|c|}
\hline \multirow{2}{*}{ Parameter } & \multicolumn{4}{|c|}{ Response } \\
\hline & & Good & Moderate & Low \\
\hline Relative BNP difference at & $\%$ & 44.3 & 24.3 & 28.6 \\
\hline 72 hours & $N$ & $N=31$ & $N=17$ & $N=20$ \\
\hline Difference of QRS filtered & $\%$ & 15.7 & 30.0 & 27.1 \\
\hline duration at 72 hours & $N$ & $N=11$ & $N=21$ & $N=19$ \\
\hline
\end{tabular}

\section{Results}

3.1. Demographics. Descriptive variables are presented in Table 1. The population consisted mainly of male participants $(82.9 \%)$, not allowing to perform adequate sex-related statistical analysis. Regarding the aetiology of the heart failure, we observed a population with $64.3 \%$ suffering from coronary artery disease and with $35.7 \%$ from nonischemic dilated cardiomyopathy.

BNP mean baseline value was $1105 \mathrm{pg} / \mathrm{mL}$, confirming a study population with severe heart failure with low mean ejection fraction at baseline $(33.5 \pm 6.3 \%)$ and high mean enddiastolic left ventricular diameter $(6.81 \pm 0.81 \mathrm{~mm})$. Mean $P$ wave was elevated at baseline $(180 \pm 9.5 \mathrm{msec})$ and mean QRS duration filtered $(149 \pm 4.1 \mathrm{msec})$ was quite higher than normal range, even if bundle branch block was present.

3.2. Neurohormonal Response. Relative BNP difference and QRS duration filtered at 72 hours are shown below (Table 2).

It is obvious by the data derived that using the specific infusion pattern, there was a $68.6 \%$ of good or moderate BNP and a $45.7 \%$ of good or moderate QRS response.

Considering the two main end-point parameters overall, there was a statistically significant BNP decrease at 48 hours after baseline $(P<.001)$, which continued at 72 hours, and a significant decrease of QRS filtered duration at 72 hours $(P=$ $.04)$. (Figures 1 and 2). However, crosstabulation of the QRS duration and the BNP response did not show any statistically significant correlation $(P>.1)$.

There were established three subgroups depending on the duration of infusion of 24 hours $(n=14), 48$ hours $(n=35)$, or 72 hours $(n=21)$.

3.2.1. Levosimendan Infusion of $24 \mathrm{~h}$. The 24-hour infusion subgroup had a mean percentage relative difference on BNP

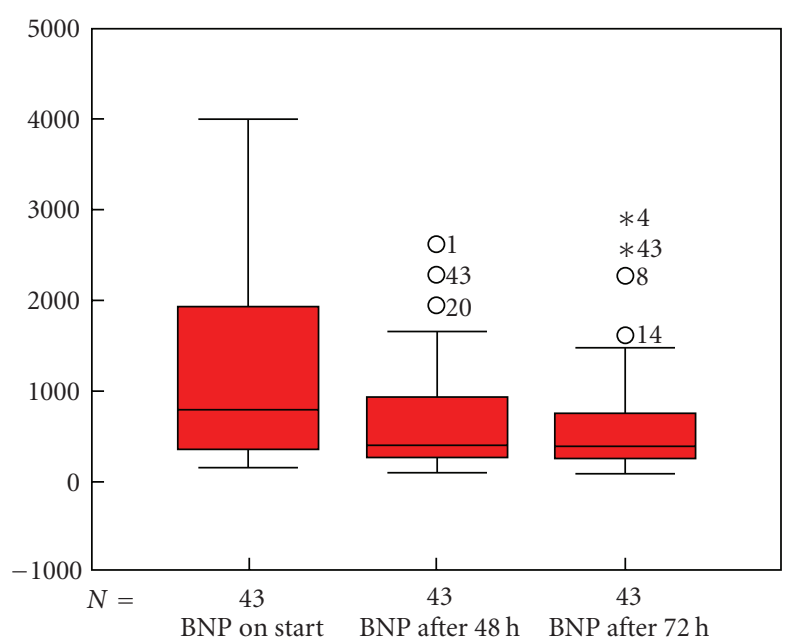

Mean \pm St. dv.: $1104 \pm 124 \quad 656 \pm 96 \quad 554 \pm 67$ $(\mathrm{pg} / \mathrm{mL})$

Figure 1: Changes of BNP after onset of levosimendan.

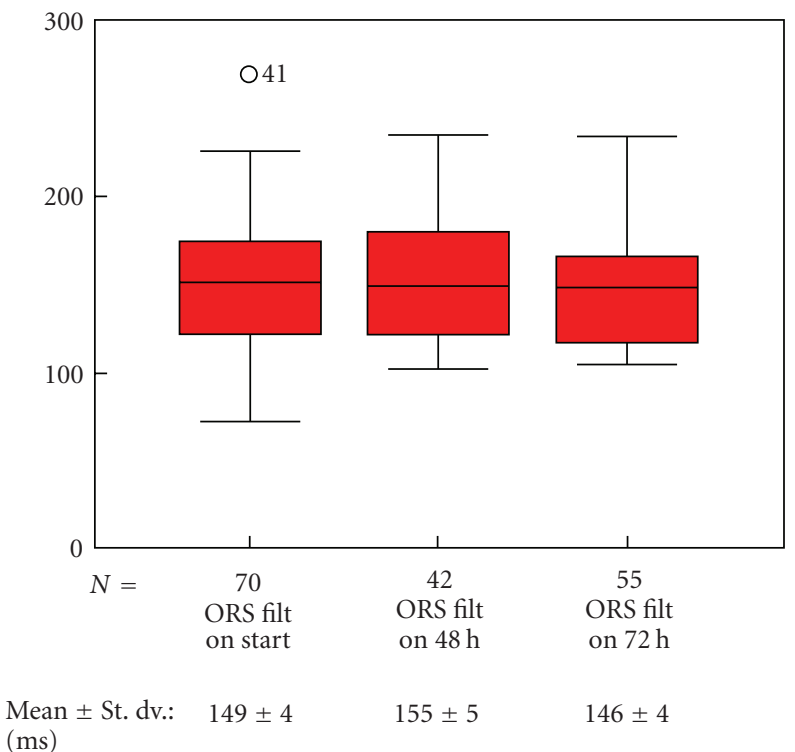

FIGURE 2: Changes of filtered QRS duration after onset of levosimendan.

at 72 hours of $15.3 \%$ and mean difference on QRS duration at 72 hours of $0.8 \mathrm{msec}$ per patient. There were no statistical significance in any measured parameter $(P>.1)$ according to the Wilcoxon test for paired differences (Table 3 ).

3.2.2. Levosimendan Infusion of $48 \mathrm{~h}$. Patients with 48-hour infusion had a statistically significant decrease on BNP beginning at 48 hours $(P<.0001)$, which continued at 72 hours $(P<.0001)$.

This finding indicates an enormous neurohormonal response with 48-hour infusion of levosimendan without loading dose. The mean percentage relative difference of BNP at 72 hours showed a decrease of $43 \%$, which is a 
TABle 3: Demographic statistics of the three subgroups (24h, 48h, and $72 \mathrm{~h}$ infusion).

\begin{tabular}{|c|c|c|c|c|}
\hline & Infusion & Start & $48 \mathrm{~h}$ & $72 \mathrm{~h}$ \\
\hline \multirow{3}{*}{$\begin{array}{l}\text { BNP- } \\
\text { Concentration } \\
(\mathrm{pg} / \mathrm{mL})\end{array}$} & 24 h-Group & $1114 \pm 320$ & $\begin{array}{c}494 \pm 101 \\
(P>.1)\end{array}$ & $\begin{array}{c}545 \pm 95 \\
(P>.1)\end{array}$ \\
\hline & 48 h-Group & $1215 \pm 193$ & $\begin{array}{c}748 \pm 151 \\
(P<.0001)\end{array}$ & $\begin{array}{c}568 \pm 104 \\
(P<.0001)\end{array}$ \\
\hline & 72 h-Group & $912 \pm 159$ & $\begin{array}{l}601 \pm 18 \\
(P<.04)\end{array}$ & $\begin{array}{c}535 \pm 29 \\
(P=.004)\end{array}$ \\
\hline \multirow{3}{*}{$\begin{array}{l}\text { QRS Duration } \\
\text { (msecs) }\end{array}$} & 24 h-Group & $151 \pm 6$ & $\begin{array}{c}159 \pm 12 \\
(P>.1)\end{array}$ & $\begin{array}{l}169 \pm 5 \\
(P>.1)\end{array}$ \\
\hline & 48 h-Group & $145 \pm 6$ & $\begin{array}{l}159 \pm 8 \\
(P>.1)\end{array}$ & $\begin{array}{c}140 \pm 6 \\
(P=.06)\end{array}$ \\
\hline & 72 h-Group & $153 \pm 8$ & $\begin{array}{c}145 \pm 10 \\
(P>.1)\end{array}$ & $\begin{array}{c}147 \pm 6 \\
(P=.05)\end{array}$ \\
\hline
\end{tabular}

Data are presented as Mean \pm Standard Deviation.

quite acceptable percent. QRS duration had on average a borderline significant decrease at 72 hours of -10.96 msecs per patient $(P=.065)$, which might indirectly indicate a decrease of left ventricular dimensions.

3.2.3. Levosimendan Infusion of $72 \mathrm{~h}$. Patients with 72-hour infusion had a borderline significant decrease on BNP beginning at 48 hours $(P=.039)$ and a statistically significant decrease at 72 hours $(P<.004)$. This finding shows a good neurohormonal response with 48-hour infusion of levosimendan without loading dose with the necessity to follow 24-hour infusion additionally, so that the decrease could achieve a significant range. Mean percentage relative difference of BNP at 72 hours was 31\%. QRS filt duration had on average a borderline significant decrease at 72 hours of -7.4 msecs per patient $(P=.049)$.

Trying a general correlation between the countable parameters of difference of QRS filtered duration at 72 hours and BNP relative difference at 72 hours, there was a linearity only in cases with adverse response for the two variables $(R$-squared $=0.01)$. Crosstabulation analysis of the two 48and 72-hour infusion patterns demonstrated that at 48-hour infusion there was an obvious correlation between good QRS responders and good BNP responders ( $P$ value of $\chi$ square test $=.05$ ). This statistical result was determined by the respective adjusted residuals of the data analysis.

At 72-hour infusion, the correlation was biased with non-BNP responders to have also a moderate QRS filtered duration response $(P>.1)$.

According to the Kolmogorov-Smirnov test and the Mann-Whitney analysis, the demographics of two subgroups of the population were compared. Without differences on the characteristics, those who have taken 48-hour infusion demonstrated at least good or moderate QRS filtered duration - at acceptable BNP_response, representing a subpopulation with good neurohormonal profile (Figure 3 ).

3.3. Safety. As for the complications, there were not patients who had to discontinue the study due to hypotension or other minor complication. Two patients died due to

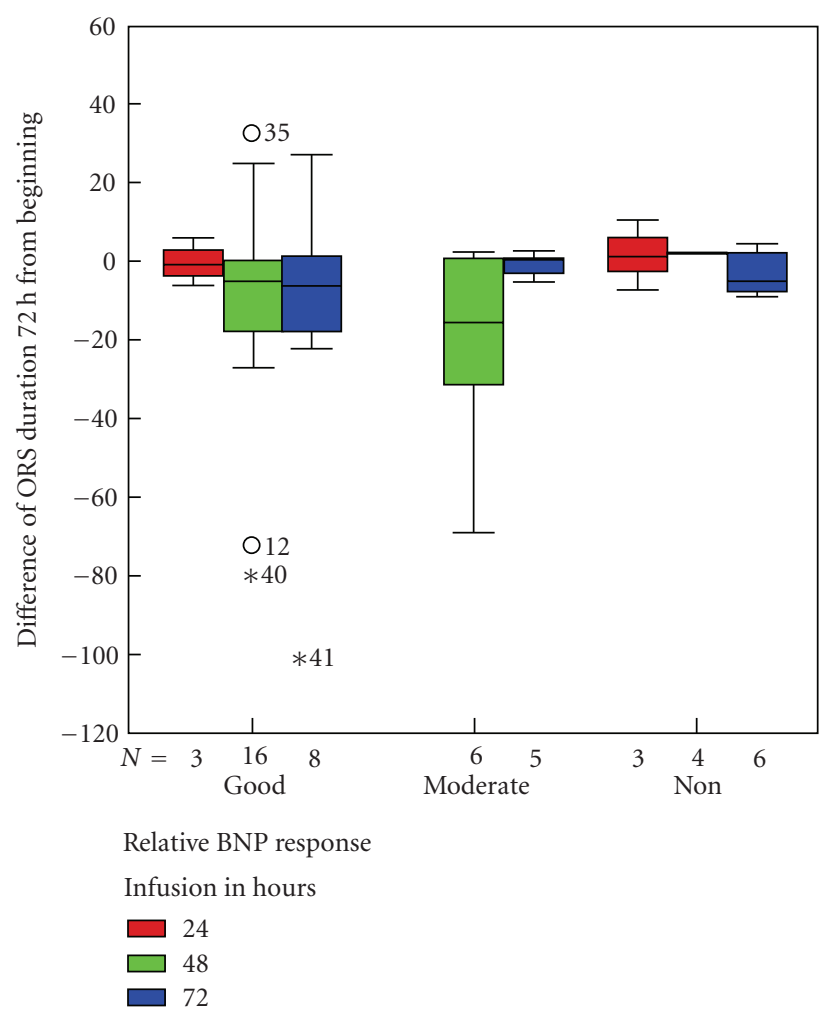

FIGURE 3: Differences of BNP response and filtered QRS duration between infusion groups.

advanced heart failure (asystole without successful reanimation). New onset of atrial flutter, atrial fibrillation or ventricular tachycardia was not observed, indicating a study population with low short-term arrhythmogenic profile according to the continuous ECG monitoring.

All patients survived achieved a weaning status by inotropic agents without the necessity of withdrawal until their discharge. Within a period of a month, one patient was presented with acute renal exacerbation, and three others needed to be rehospitalised for exacerbated heart failure.

\section{Discussion}

In this study, we tried to demonstrate efficiency and safety of an alternative infusion pattern of levosimendan in acute heart failure. The critically ill patients of this condition have most of the time a borderline preserved systolic pressure. So, it is important to prevent the patient from hypotension, which could induce hypoperfusion. Levosimendan has on start an enormous peripheral vasodilatory effect, which causes hypotension [9].

In this view, participants were not given the loading dose at the beginning, but a continuous dose was administrated up to 72 hours depended on the clinical response.

$\mathrm{BNP}$ is widely recognized as a therapeutic tool of responsiveness in patients with heart failure [10]. In our study, we used BNP as a reliable marker of efficiency [11]. 
It was obvious from the data analysis that those patients who were given 72-hour infusion had the necessity for such a long infusion. They did not achieve clinical improvement at 48 hours, so they had to continue with another 24hour infusion. The population studied were patients with advanced heart failure, since mean value was generally above $1000 \mathrm{pg} / \mathrm{mL}$. This means that the percent of decrease of BNP was, respectively, not so enormous like in other trials [12], in which the mean value was about $500-800 \mathrm{pg} / \mathrm{mL}$. We think that the achieved mean values of $31-43 \%$ are acceptable improvement [13], concerning that all patients survived did not have symptoms or re-exacerbation of their clinical status.

The other basic bound of our study was the investigation of safety of such infusion patterns. As for the complications observed, there was no indication that we had arrhythmogenic effect of the agent. The two deaths were due to advanced heart failure and could be accepted by the condition of acute heart failure [14].

The signal averaged ECG is an examination which has a good specificity in predicting monomorphic ventricular tachycardia in patients with chronic heart failure. Its power is obvious in patients after myocardial infarct [15]. There are also studies with patients of dilated cardiomyopathy [16], which mention the role of signal-averaged ECG. However, in the last years, the role of this examination has diminished [17].

The scope was to show that levosimendan did not at least change the parameters of this examination, so that a neutral effect could be confirmed. Indeed, the study showed that in good BNP responders there was a subpopulation whose QRS filtered duration not only was unchanged but also decreases. The finding of this observation cannot be evaluated exactly but indicates a subpopulation with good clinical and neurohormonal response. Further studies should be conducted to investigate the correlation between BNP and filtered QRS duration variations.

Considering the results of this study, it could be said that levosimendan is an inotropic drug, which allows an infusion of more than 24 hours. There is also an opposed opinion [18], which supports that there is no need of prolonged infusions, since the metabolites of levosimendan have long half-life period. The problem is that sometimes patients admitted are critically ill and the target of treating them efficiently is difficult to achieve.

We need an inotropic agent like levosimendan with an acceptance of $\beta$-blocker coadministration, or at least at low doses, with non- $\beta$-agonist effect and energetically beneficial. Furthermore, at this clinical condition, a druginduced hypotension could be unnecessary. So, patterns without loading dose could be beneficial.

Limitations of the study are its design, which does not have perspective analysis and randomization of groups in an objective manner. It is an observational study with the additional limitation of small period of followup of the variables measured.

Another limitation is the absence of hemodynamic data $[19,20]$, which could support adequately the scope of the study. This could be the goal of a future study in the same way of using alternative infusion patterns.
The subgroup observed in this study with the beneficial profile should be followed in time with the hope to stratify the cumulative risk of mortality or morbidity in heart failure [21].

\section{Acknowledgment}

G. Aidonidis (University of Duisburg-Essen, Germany; Aristotle University of Thessaloniki, Greece), I. Kanonidis (Aristotle University of Thessaloniki, Greece), V. Koutsimanis (Aristotle University of Thessaloniki, Greece), T. Neumann (University of Duisburg-Essen, Germany), R. Erbel (University of Duisburg-Essen, Germany), and G. Sakadamis (Aristotle University of Thessaloniki, Greece) are all on behalf of the Competence Network of Heart Failure.

\section{References}

[1] A. Lehmann, J. Boldt, and J. Kirchner, "The role of Ca++sensitizers for the treatment of heart failure," Current Opinion in Critical Care, vol. 9, no. 5, pp. 337-344, 2003.

[2] M. S. Nieminen, J. Akkila, G. Hasenfuss et al., "Hemodynamic and neurohumoral effects of continuous infusion of levosimendan in patients with congestive heart failure," Journal of the American College of Cardiology, vol. 36, no. 6, pp. 1903$1912,2000$.

[3] R. Takahashi, M. A. H. Talukder, and M. Endoh, "Inotropic effects of OR-1896, an active metabolite of levosimendan, on canine ventricular myocardium," European Journal of Pharmacology, vol. 400, no. 1, pp. 103-112, 2000.

[4] M. Sharma and J. R. Teerlink, "A rational approach for the treatment of acute heart failure: current strategies and future options," Current Opinion in Cardiology, vol. 19, no. 3, pp. 254-263, 2004.

[5] M. Packer, "REVIVE II. Metanalysis-Selected adverse events," American Heart Association. Scientific Sessions, 2005.

[6] A. Nohria, S. W. Tsang, J. C. Fang et al., "Clinical assessment identifies hemodynamic profiles that predict outcomes in patients admitted with heart failure," Journal of the American College of Cardiology, vol. 41, no. 10, pp. 1797-1804, 2003.

[7] A. Gegenhuber, T. Mueller, F. Firlinger, K. Lenz, W. Poelz, and M. Haltmayer, "Time course of B-type natriuretic peptide (BNP) and N-terminal ProBNP changes in patients with decompensated heart failure," Clinical Chemistry, vol. 50, no. 2, pp. 454-456, 2004.

[8] S. Mavrogeni, G. Giamouzis, E. Papadopoulou et al., "A 6month follow-up of intermittent levosimendan administration effect on systolic function, specific activity questionnaire, and arrhythmia in advanced heart failure," Journal of Cardiac Failure, vol. 13, no. 7, pp. 556-559, 2007.

[9] P. Põder, J. Eha, S. Sundberg et al., "Pharmacodynamics and pharmacokinetics of oral levosimendan and its metabolites in patients with severe congestive heart failure: a dosing interval study," Journal of Clinical Pharmacology, vol. 44, no. 10, pp. 1143-1150, 2004.

[10] A. S. Maisel et al., "The diagnosis of acute congestive heart failure: role of BNP measurements," Heart Failure Reviews, vol. 8, no. 4, pp. 327-334, 2003.

[11] G. Giamouzis, G. Giannakoulas, K. Tsarpalis et al., "Natriuretic peptide-guided levosimendan therapy for heart failure: a promising new approach," International Journal of Cardiology, vol. 128, no. 1, pp. 91-93, 2008. 
[12] P. Arruda et al., "Long term effect of levosimendan upon BNP levels in patients with decompensated heart failure," Critical Care Medicine, vol. 32, no. 12, supplement, 2004.

[13] G. Gianakoulas, G. Giannoglou, V. Vassilikos et al., "Clinical significance of acute neorohormonal respons after levosimendan treatment," American Journal of Cardiology, vol. 98, pp. 1123-1124, 2006.

[14] M. S. Nieminen, M. Böhm, M. R. Cowie et al., "Executive summary of the guidelines on the diagnosis and treatment of acute heart failure: the Task Force on Acute Heart Failure of the European Society of Cardiology," European Heart Journal, vol. 26, no. 4, pp. 384-416, 2005.

[15] American College of Cardiology Expert Consensus Document, "Signal- averaged Electrocardiography," Journal of the American College of Cardiology, vol. 27, no. 1, pp. 238-249, 1996.

[16] D. Provan and A. Krentz, Eds., Oxford Handbook of Clinical and Laboratory Investigation, Oxford University Press, Oxford, UK, 2003.

[17] L. Toivonen, M. Viitasalo, S. Sundberg, J. Akkila, and L. Lehtonen, "Electrophysiologic effects of a calcium sensitizer inotrope levosimendan administered intravenously in patients with normal cardiac function," Journal of Cardiovascular Pharmacology, vol. 35, no. 4, pp. 664-669, 2000.

[18] L. De Luca, W. S. Colucci, M. S. Nieminen, B. M. Massie, and M. Gheorghiade, "Evidence-based use of levosimendan in different clinical settings," European Heart Journal, vol. 27, no. 16, pp. 1908-1920, 2006.

[19] J. Lilleberg, M. Laine, T. Palkama, M. Kivikko, P. Pohjanjousi, and M. Kupari, "Duration of the haemodynamic action of a 24-h infusion of levosimendan in patients with congestive heart failure," European Journal of Heart Failure, vol. 9, no. 1, pp. 75-82, 2007.

[20] J. G. F. Cleland, N. Freemantle, A. P. Coletta, and A. L. Clark, "Clinical trials update from the American Heart Association: REPAIR-AMI, ASTAMI, JELIS, MEGA, REVIVEII, SURVIVE, and PROACTIVE," European Journal of Heart Failure, vol. 8, no. 1, pp. 105-110, 2006.

[21] A. Varro and J. G. Papp, "Classification of positive inotropic actions based on electrophysiologic characteristics: where should calcium sensitizers be placed?" Journal of Cardiovascular Pharmacology, vol. 26, no. 1, pp. S32-S44, 1995. 


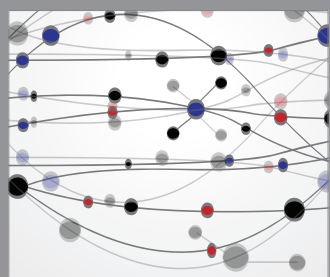

The Scientific World Journal
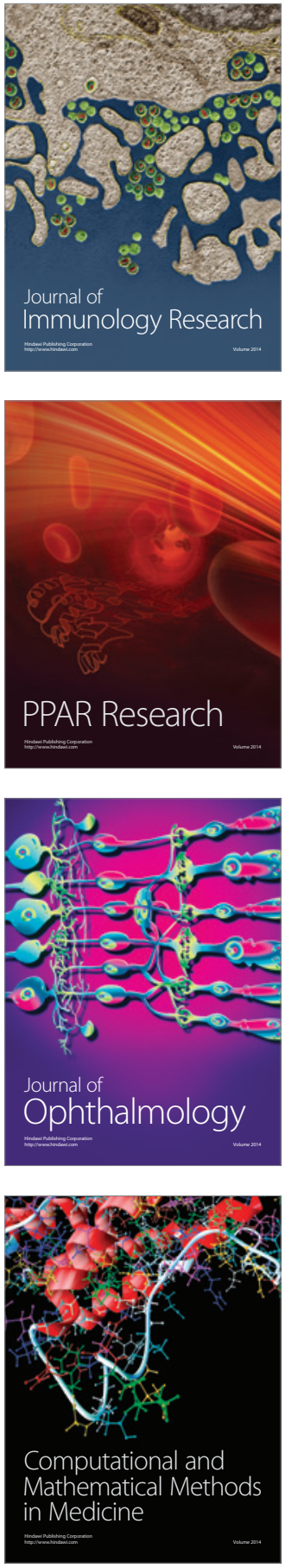

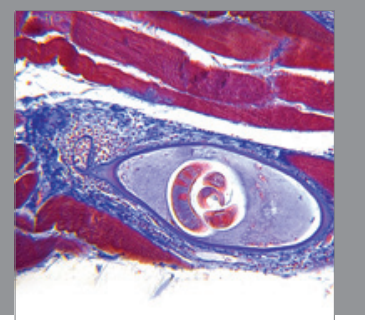

Gastroenterology

Research and Practice
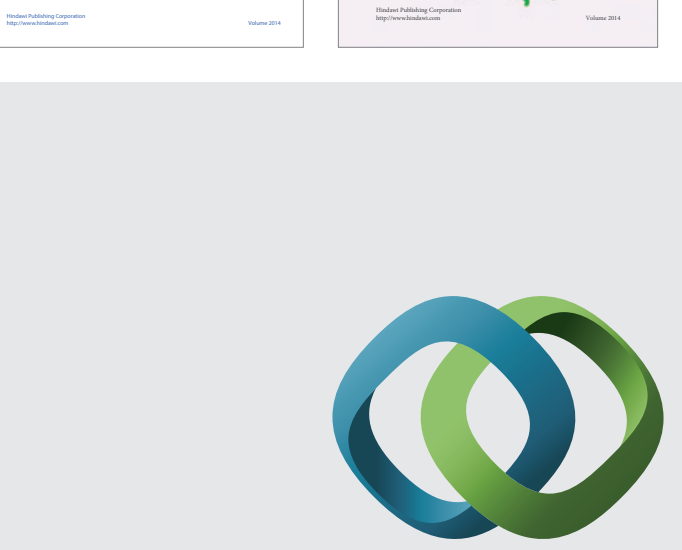

\section{Hindawi}

Submit your manuscripts at

http://www.hindawi.com
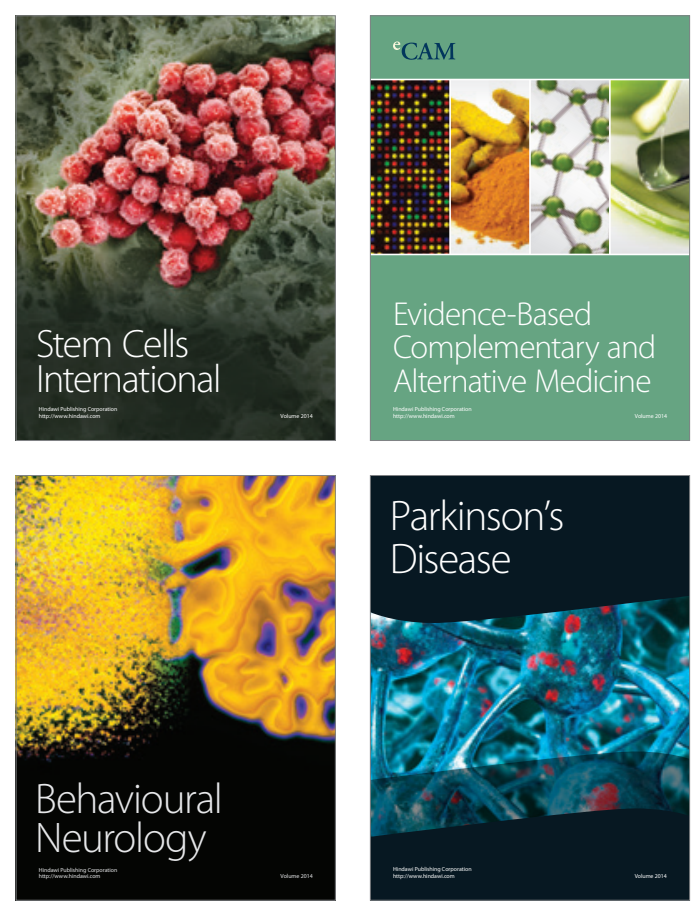

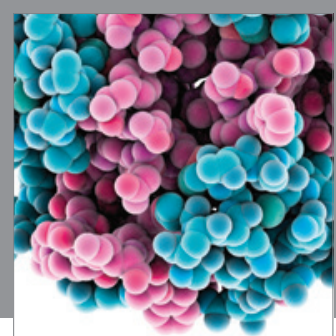

Journal of
Diabetes Research

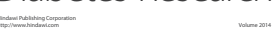

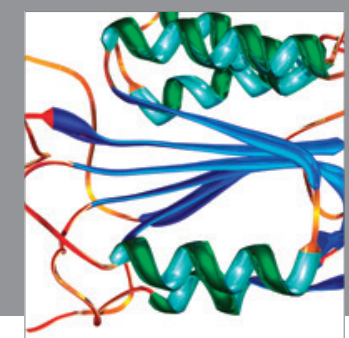

Disease Markers
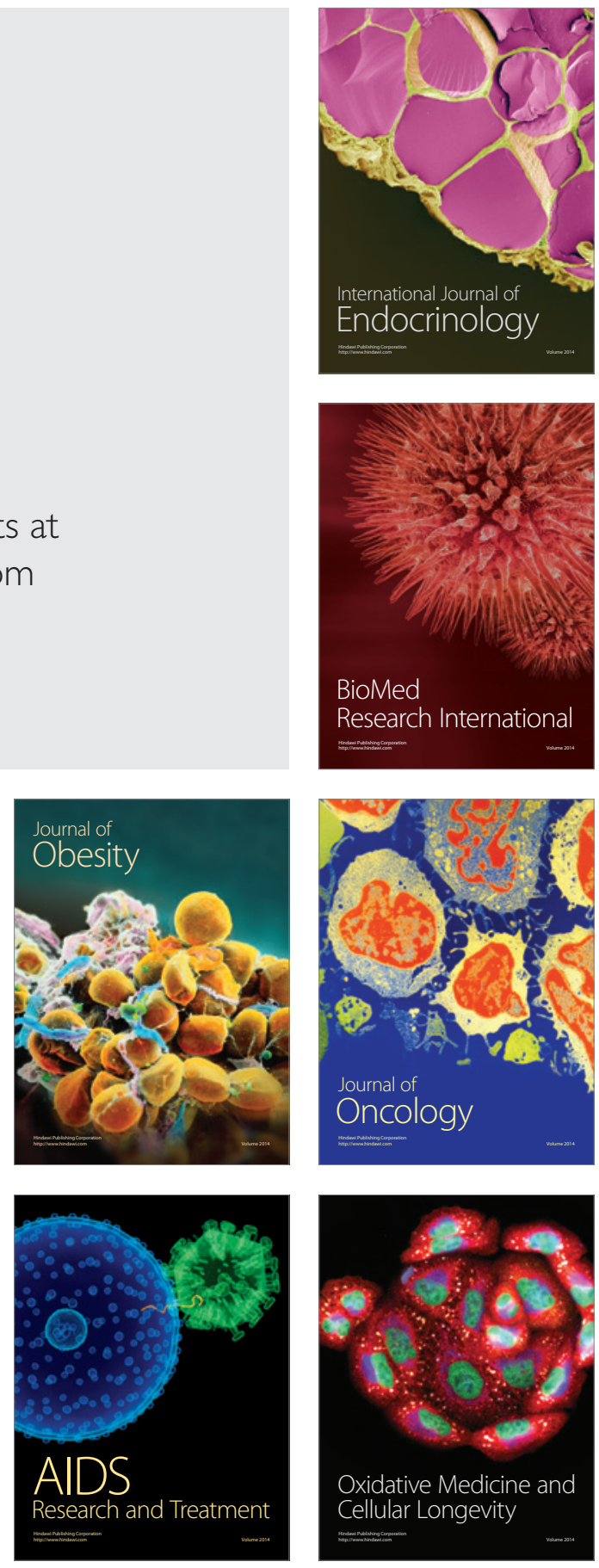